\title{
Gestão do conhecimento, tecnologia da informação e pequenas e médias empresas de serviços: um estudo de casos múltiplos na Região Metropolitana do Recife
}

\author{
André Felipe Albuquerque Fell ${ }^{I}$ \\ http://orcid.org/0000-0002-3179-2595 \\ Jairo Simão Dornelas ${ }^{I I}$ \\ I Universidade Federal de Pernambuco, PE, Brasil. \\ Docente no Departamento de Ciência da Informação. \\ ${ }^{I I}$ Universidade Federal de Pernambuco, PE, Brasil. \\ Docente no Programa de Pós-graduação em Administração.
}

http://dx.doi.org/10.1590/1981-5344/3781

Pode-se afirmar que a gestão do recurso intangível demanda uma nova compreensão e esforço para relacionar os fatores organizacionais como estrutura, estratégia, processo, pessoas e tecnologia. Tal fato deveria ser considerado pelas pequenas e médias empresas - PME. Olhando este panorama, a presente pesquisa visa verificar quais são os fatores organizacionais obstativos ao uso da tecnologia da informação - TI - para a gestão do conhecimento em PME de serviços da Região Metropolitana do Recife. Para tal, optou-se pela abordagem de pesquisa qualitativa, sendo os dados coletados por cinco extensas entrevistas semiestruturadas e analisados de modo interpretativo pela técnica de análise de conteúdo. Constatou-se na realidade vivenciada pelas PME que a gestão do conhecimento 
ainda é futuro, é meta que talvez venha a ser atingida mediante melhor entendimento e concepção da prática de gestão da informação e o uso da TI ainda é essencialmente operativo e com fins de organizar, sistematizar e tornar eficiente as atividades cotidianas estando mui longe da gestão do conhecimento.

Palavras-chave: Gestão do conhecimento; Tecnologia da informação; Pequenas e médias empresas de serviços;Região Metropolitana do Recife.

\section{Knowledge management, information technology, small and medium sized service enterprises: a multiple case study at Recife urban region}

The intangible resource managementrequires a new comprehension and struggle to relate organizational key factors likestructure, strategy, process, people and technology. That is something small and mediumsized enterprises (SMEs) ought to pay attention to.The present research has the mainpurpose to check what are the key organizational factors that turned to be obstacles to theuse of IT for knowledge management in small and medium sized service enterprises at Recife urban region. Therefore a qualitative research method hasbeen chosen and data has been collected by a five extensive semistructured interview script. Data has been analyzed in an interpretative way, using content analysis. In the small and mediumsized service enterprises' reality it was possible to notice that knowledge management is a futureproject, a goal that may be achieved by a better comprehension and conception ofinformation management practices. Also the IT use is still mainlyoperational and has a central function in organizing efficiently activities and in this sense isstill quite distant from knowledge management. 
Keywords: Knowledge management; Information technology; Small and medium sized service enterprises; Recife urban region.

Recebido em 14.11.2018 Aceito em 13.05.2020

\section{Introdução}

Um dos aspectos perceptíveis de mudança que a tecnologia da informação (TI) desencadeou quer na sociedade quer nas organizações de pequeno, médio ou grande porte foia mudança no comportamento dos indivíduos que passaram a se comunicar eletronicamente através de padrões computacionais abertos e universais (EVAN; WURSTER, 1997), induzindo um aumento de interconectividade que possivelmente representa o fator de maior revolução para as estratégias de negócios, além de significar um aumento no fluxo de informações na realidade das organizações (CUNHAet. al., 2008). Daí Davenport (2012) observar que progressivamente as organizações estão tendo que lidar com uma expansão de dados volumosos e desestruturados que não mais podem ser gerenciados e analisados por meios tradicionais, mas demandam novas formas de análise e processamento de tais dados de modo a gerar informações oportunas e pertinentes.

Este aumento crescente de fluxo, difusão e manipulação da informação, afetando a organização tanto nas suas relações externas quanto nas relações internas é uma das mudanças decorrentes da economia da informação que está ligada tanto à informação quanto à sua tecnologia associada (SHAPIRO; VARIAN, 1999). Assim, a tecnologia da informação passou a não ter mais simplesmente o caráter de apoio para a organização, passando a ser encarada como recurso estratégico crítico (PRAHALAD; RAMASWAMY, 2004); permitindo ganho de produtividade alcançado pelos resultados da automação dos processos na produção ou gestão (MURPHY; SAMIR, 2009; KESS et. al., 2010).

Mas para que a TI possa ter um papel estratégico para as organizações é preciso que os objetivos e interesses da organização estejam alinhados às plataformas tecnológicas disponíveis (ALBANO, 2001). Isto significa que para a implantação bem sucedida de uma tecnologia da informação, as organizações devem apropriar-se de seus benefícios, integrando-a a outras ferramentas de gestão o que, por conseguinte, gerará benefícios realmente significativos a médio e longo prazo (SILVA; FISCHMANN, 2002).

Além disso, é importante a realização de uma análise mais abrangente que diz respeito aos impactos na eficácia organizacional da seleção de aplicações de tecnologia da informação, não se restringindo 
aos aspectos técnicos de informática e nem aos aspectos de funcionalidade da aplicação (MORAES, 2005). É preciso avaliar os eventuais resultados obtidos, tendo uma clara visão estratégica do alinhamento da TI com as estratégias das áreas do negócio para que se consiga identificar a extensão dos impactos nos resultados obtidos e os ganhos de produtividade e competitividade do negócio como medida de eficácia organizacional (WALTON, 1993; LAURINDO et al., 2002; KISSIMOTO, 2011).

Há fortes evidências indicando que a eficiente gestão de recursos de conhecimento organizacional é um aspecto vital para a melhoria da competitividade empresarial (EGBU; BOTTERILL, 2001; KAMARA et. al., 2002; QUINTAS, 2002; HUANG et. al., 2011; MAGNIER-WATANABE; BENTON; SENOO, 2011), daí ser razoável considerar que para as grandes, pequenas e médias empresas terem condições de sobreviverem em uma economia extremamente competitiva e global, precisarão cuidar da gestão de seu conhecimento organizacional. Para tal intento, a TI pode desempenhar papel decisivo ao oportunizar as ferramentas que possibilitam uma eficiente gestão do conhecimento (RUGGLES, 1997; ANGUS; PATEL; HARTY, 1998; JACKSON, 1999; WENSLEY, 2000; TYNDALE, 2002).

Ademais, considerando a importância das pequenas e médias empresas - PME- na economia de países industrializados (GELINAS; BIGRAS, 2004) e na economia brasileira (CALADO, 2004; IBGE, 2010), as características e especificidades deste segmento de empresas em relação à realidade das grandes empresas (BORTOLI NETO, 1980; VIDAL, 1990; KASSAI, 1996; THONG, 2001), torna-se importante o estudo destas empresas quanto ao uso que fazem da tecnologia da informação em suas atividades diárias de gerenciamento do conhecimento.

Entretanto, há que se considerar, que a dificuldade que as PME têm em assimilar tecnologias da informação é um fator preocupante, uma vez que a sua eficaz utilização pode tornar o seu ambiente de atuação mais produtivo por possibilitar um aprimoramento tanto na coleta de dados quanto na geração de informações, melhorando sua competitividade e, por conseguinte, a sua lucratividade (ZIMMERER; SCARBOROUGH, 1994; LUNARDI; DOLCI; MAÇADA, 2010; SOUZA; ARPINO, 2011).

É possível afirmar que há pesquisas desenvolvidas sobre gestão do conhecimento em PME (BEIJERSE, 2000; LIM; KLOBAS, 2000; FREY, 2001; HENG, 2001; KAUTZ; THAYSEN, 2001; WICKERT, HERSCHEL, 2001; WONG, 2005), contudo, poucas foram as pesquisas nacionais identificadas que buscaram estudar o uso de TI por parte das PMEs (SILVA, 2002; FELL, 2003; MORAES et. al., 2004; PRATES; OSPINA, 2004; LUNARDI; DOLCI; MAÇADA, 2010; NGANGA; LEAL, 2013; SIQUEIRA; SOUZA; CHAGAS, 2014)e até o presente momento, foram 
identificados apenas dois estudos nacionais que enfatizaram a gestão do conhecimento em pequenas e médias empresas (KRUGLIANSKAS; TERRA, 2004; FELL, 2009).

Praticamente não foi encontrado nenhum estudo abordando os fatores que obstaculam as PME de usarem a TI para a gestão do conhecimento. Nesta conjuntura, pretende-se investigar o contexto problemáticomediante a seguinte questão de pesquisa: quais são os fatores organizacionais que na realidade vivenciada por pequenas $e$ médias empresas de serviços na Região Metropolitana do Recife (RMR) obstaculam o uso da tecnologia de informação para a gestão do conhecimento?

Justifica-se a realização da incursão em campo dada à vitalidade do segmento PME para a atividade econômica nacional e regional e também e pela especificidade de atuação da $P M E$, quase sempre focada na competência de um ou poucos indivíduos para realizarem um trabalho focado.

A seguir traça-se um breve espelho da malha de conceitos essencial à pesquisa.

\section{Gestão do conhecimento}

A perspectiva do conhecimento como recurso econômico, além de ter gerado muitos debates, livros e conferências em nível mundial desde a última década do século passado, vem refletindo as rápidas mudanças sociais, econômicas e tecnológicas, que desde a década de 1970 vêm influenciando a vida dos indivíduos e das organizações (SOUZA; HENDRIKS, 2006). Nas organizações, estas mudanças têm desencadeado modificações em práticas e processos organizacionais, particularmente nas áreas que dependem de competências individuais ou coletivas. Tais modificações internas podem ser compreendidas pelo fato do conhecimento estar cada vez mais desempenhando um importante papel no turbulento e competitivo mercado atual, induzindo as organizações a atentarem para a necessidade de se gerenciá-lo de forma efetiva (SEDIGHI; MOKFI; GOLRIZGASHTI, 2012), particularmente nos umbrais das organizações (GONZALEZ; MARTINS; MELO, 2018).

O esforço por promover o conhecimento nas organizações acabou convergindo para o conceito de gestão do conhecimento - GC- que encontrou apoio tanto no ambiente acadêmico quanto no empresarial.Este apoio veio caracterizado na diversidade de estudos, quer mostrando o valor ou a localização do conhecimento organizacional quer empreendendo um esforço de sua criação, desenvolvimento, compartilhamento e difusão (SOUZA; HENDRIKS, 2006; FREIRE; SPANHOL, 2014).

Em seus estudos, Hautala (2011) identificou que o gerenciamento do conhecimento, dentro das organizações, está voltado para melhorar o 
desempenho econômico e social dos países, unindo-se às contribuições das universidades. Daí Rosario, Kipper e Frozza (2014) defenderem a necessidade de se desenvolver formas e metodologias para gerenciar o conhecimento como sendo vital para a determinação da vantagem competitiva de uma organização; levando Leonardi e Bastos (2014) a notarem que o conhecimento vem apresentando destaque crescente e relevante dentro das organizações contemporâneas.

Acontece que para Swan e Scarbrough (2001), foi apenas no fim do século XX que a gestão do conhecimento começou a ser pronunciada como discurso gerencial. Esta perspectiva evoluiu a partir de uma visão organizacional embasada em recursos, na qual a diversidade, qualidade e inovação dos recursos internos proporcionavam uma base melhor, mais flexível e duradoura para a definição de estratégias do que os produtos ou serviços gerados destes recursos.

Assim, por ser considerada uma disciplina nova no campo da administração, a gestão do conhecimento ainda hoje é objeto de uma diversidade de abordagens, definições e percepções (STOLLENWERK, 2001). Independentemente da definição adotada, a noção de conhecimento como recurso e fonte de estratégia competitiva, já despontara com a virtude de queo mesmo deveria ser gerenciado mais judiciosamente, mais eficientemente e de forma sistemática (QUINTASet. al., 1997).

Com esta ação, admite-se que a gestão do conhecimento direciona políticas, estratégias e técnicas que dão suporte à competitividade organizacional por meio da otimização das condições necessárias à melhoria na eficiência, inovação e colaboração entre funcionários (NONAKA; TAKEUCHI, 1997; ZACK, 1999; TEECE, 2000). Autores e praticantes da gestão do conhecimento têm procurado desenvolver abordagens gerais de intervenção, tanto organizacional quanto tecnológica, que são centradas em promover a efetividade de processos de conhecimento, tais como a criação, desenvolvimento, difusão, compartilhamento e proteção do conhecimento (DAVENPORT; PRUSAK, 1998; PROBST; RAUB; ROMHARDT, 2002). Porém, a temática gestão do conhecimento é considerada modismo para alguns estudiosos (WENGER, 1998; SWANet. al., 1999; 2001).

No presente estudo, a gestão do conhecimento será entendida como "o esforço sistemático e intencional da organização de procurar desenvolver atividades responsáveis por gerar, transferir, compartilhar, armazenar e utilizar de forma eficiente o conhecimento que circula dentro dela, de modo a poder incorporá-lo às estratégias, sistemas, processos, decisões, produtos e serviços"(FELL, 2011, p.45). 
E sua a massificação, caso exista, direcionada ao cenário de PME (WILLERDING, 2015).

\section{Tecnologia da informação para a gestão do conhecimento}

Pode-se definir a tecnologia da informação como "o conjunto de recursos não-humanos empregados na coleta, armazenamento, processamento e distribuição da informação" (AUDY; ANDRADE; CIDRAL, 2005, p. 187). Este instrumento auxilia os gestores no processo decisório quando estes se deparam com um volume de dados desestruturados oriundos de várias fontes, como a Internet, celulares, portais sociais dentre outros (DI MARTINO et. al., 2014).

Em outros termos, a TI é a infraestrutura que permite armazenar, buscar, recuperar, copiar, filtrar, manipular, visualizar, transmitir e receber informação (SHAPIRO; VARIAN, 1999) para a geração de alternativas para a tomada de decisão (KAHNEMAN; LOVALLO; SIBONY, 2011). Além disso, a TI abrange os métodos, as técnicas e as ferramentas para o planejamento, desenvolvimento e suporte dos processos de utilização da informação (AUDY; ANDRADE; CIDRAL, 2005).

$\mathrm{Na}$ atual conjuntura empresarial, observa-se que a TI tem significativa relevância em diversos fatores (MCGEE; PRUSAK, 1994; HARRISON et al., 1997; STÁBILE; CAZARINI, 2000; ALBERTIN, 2001; PRATES, 2002; MORAES; ESCRIVÃO FILHO, 2004; MARCHAND et al., 2004; MIAKE; CARVALHO; PINTO, 2014; FREITAS JUNIOR; MAÇADA, 2014), tais como:

- Presença em todos os processos administrativos e produtivos, apoiando o processo de inovação no desenvolvimento de novos produtos e serviços;

-Auxílio prestado na tomada de decisão e na melhoria da eficiência das operações empresariais;

- Capacidade de alterar muitas atividades gerenciais, mão-de-obra, profissões e ramos de negócios;

- Aprendizado e aperfeiçoamento da produtividade individual;

- Gerenciamento de volumes e complexidade dos dados empresariais;

- Ganho de produtividade por automatizar trabalhos repetitivos;

- Representar uma das mais poderosas influências no planejamento das organizações podendo, inclusive, colaborar com a estratégia competitiva das empresas, proporcionando vantagens competitivas.

- Facilitar a interação entre profissionais de TI e de marketing na especificação conjunta de projetos corporativos de gestão do conhecimento de clientes oCustomerKnowledge Management- CKM -. 
Desse modo, a TI tem se apresentado uma poderosa engrenagem no progresso econômico e, algumas vezes, o principal direcionador do aumento da produtividade. Daí as organizações utilizarem um variado e complexo conjunto de tecnologias no seu processo produtivo e administrativo, objetivando alcançar maiores níveis estratégicos de produtividade, uma vez que há uma maior agilidade nos processos de obtenção e manipulação das informações (XAVIER, 2003). Tal perspectiva é reiterada por Beal (2004) que considera a TI como facilitadora do acesso às fontes de conhecimento de maneira mais rápida e a um menor custo, oferecendo variadas opções para a sua criação, distribuição, recuperação e preservação.

De fato, a possibilidade de a tecnologia da informação intermediar a gestão do conhecimento é reforçada por pesquisa levada a cabo na base de artigos apresentados nos fóruns anuais promovidos pela Associação Nacional dos Programas de Pós-Graduação em Administração - ANPAD, tomando como referência o período de 1997 a 2007 (FELL; RODRIGUES FILHO; OLIVEIRA, 2008). Na pesquisa citada, foi possível constatar que em torno de $38 \%$ dos artigos sobre o tema estão ligados à área temática de Administração da Informação, isto é, uma área em que a TI é marcante.

Para Laudon e Laudon (2016), a TI desempenha papel importante na gestão do conhecimento como habilitadora de processos de negócios que objetivam criar, armazenar, disseminar e aplicar conhecimento. A visão da TI como infraestrutura para a gestão do conhecimento também já fora enunciada por Carvalho (2003), para quem o principal papel da TI na GC consistia em acelerar a velocidade de transferência do conhecimento. Apesar de ser uma importante ferramenta que possibilita a implantação da gestão do conhecimento nas organizações, a tecnologia da informação não deve ser confundida com a gestão do conhecimento propriamente dita, devendo ser considerada uma ferramenta de apoio à geração, ao armazenamento, ao controle e à difusão do conhecimento.

No presente estudo, três conjuntos de tecnologias foram analisados, de modo a contribuírem para a gestão do conhecimento em pequenas e médias empresas de serviços da Região Metropolitana do Recife - RMR:

-As tecnologias comunicativas - tais como e-mail; intranets corporativas; Internet; ferramentas baseadas na Web; e o telefone;

- As tecnologiascolaborativas - tais como os recursos de computação colaborativa (brainstormingeletrônico), tecnologias workflow, Lotus Notes $\AA$ e outras ferramentasgroupware;

-As tecnologias de armazenagem - similares aos sistemas de gestão de banco de dados (SGBD), sistemas de gestão de documentos eletrônicos e sistemas especializados de armazenagem. 


\section{Procedimentos metodológicos}

No presente estudo, optou-se pelo método de abordagem qualitativa, uma vez que estaabordagem é indicada para captar as perspectivas e interpretações das pessoas (ROESCH, 1999). Já em termos de estratégia de pesquisa optou-se pelo estudo de caso coletivo porque se tem a expectativa de que o estudo de vários casos, semelhantes ou diversos, pode melhorar o entendimento dos fenômenos (STAKE, 1994).

Para Merriam (1998), os casos coletivos recebem a denominação de casos múltiplos. Assim, quanto mais casos houver em um estudo, maior é a possibilidade de variações nos casos, tornando a interpretação mais fundamentada. Presentemente, como a pesquisa tem como objeto de estudo quatro PME de serviços da RMR, optou-se pelo estudo de caso com casos múltiplos.

$\mathrm{Na}$ fase de coleta de dados utilizou-se a técnica de entrevistas pessoais com questões abertas, ou seja, entrevistas semiestruturadas nas quais o pesquisador tem como objetivo entender a perspectiva dos participantes do estudo (ROESCH, 1999). Optou-se pela entrevista semiestruturada, pois

ao mesmo tempo em que valoriza a presença do investigador, oferece todas as perspectivas possíveis para que o informante alcance a liberdade e a espontaneidade necessárias, enriquecendo a investigação (TRIVIÑOS, 1995, p. 146).

Constituindo-se como um estudo em que o pesquisador investiga, registra, interpreta e correlaciona fatos e variáveis sem manipulá-los (MARTINS, 1994), caracterizou-se, por isso,como um estudo nãoexperimental e descritivo, no qual os dados foram analisados de forma interpretativa, utilizando-se da técnica de análise de conteúdo para buscar responder à questão de pesquisa mencionada.

\subsection{Definição das unidades de análise}

A escolha inicial das PME da RMR para a pesquisa aconteceu a partir de uma consulta ao banco de dados da mais antiga instituição (43 anos) a promover o debate e troca de experiências quanto ao uso da TI nas organizações localizadas no Estado de Pernambuco, a SUCESU-PE. A partir daí, pelo critério de acessibilidade, optou-se intencionalmente por 4 (quatro) PME do segmento econômico de serviços e que tinham de 10 a 500 funcionários.

Ressalta-se ainda que as PME escolhidas deveriam ter identificada alguma prática diária de utilização da Internet (como ferramenta de comunicação, colaboração e compartilhamento de informação ou conhecimento) e, no mínimo, usarem o correio eletrônico (e-mail) para 
alguma transação informacional cotidiana. O Quadro 1 a seguir sintetiza as PME escolhidas.

Quadro 1 Pequenas e médias empresas entrevistadas do segmento de serviços

\begin{tabular}{|c|c|c|c|c|c|}
\hline \multirow[b]{2}{*}{ EMTRESA } & \multirow{2}{*}{$\begin{array}{r}\text { ANO DE } \\
\text { rUNDAC } \\
\end{array}$} & \multirow{2}{*}{$\begin{array}{c}\mathrm{N} D E \\
\text { EMIREGAdos }\end{array}$} & \multirow{2}{*}{$\begin{array}{r}\text { TIPO DE } \\
\text { NEGOCIO } \\
\end{array}$} & \multicolumn{2}{|c|}{$\begin{array}{c}\text { NF DE PESSOAS POR }_{\text {GRUPOS }} \\
\text { GRUS }\end{array}$} \\
\hline & & & & GRI & GR2 \\
\hline $\mathrm{A}$ & 1940 & 301 & Faculdade de Ensino Superior Privado & 01 & 01 \\
\hline $\mathrm{E}$ & 2003 & 80 & Serviço de Gráfica e Editoração & 01 & - \\
\hline $\mathrm{G}$ & 1989 & 150 & $\begin{array}{l}\text { Serviço de Desenvolvimento de } \\
\text { Software voltado para o Varejo }\end{array}$ & 01 & - \\
\hline $\mathrm{L}$ & 1998 & 174 & $\begin{array}{c}\text { Serviço de Tecnologia da Informaçäo e } \\
\text { Engenharia }\end{array}$ & 01 & - \\
\hline
\end{tabular}

Fonte: Dados coletados na pesquisa de campo.

Fora projetado dividir as entrevistas em 3 (três) grupos: gerencial, técnico e administrativo. Contudo, na fase de coleta foram entrevistadas quatro pessoas ocupando cargos gerenciais e uma pessoa ocupando o cargo técnico. Tal fato se justifica pelo fato de o pesquisador ter tido maior acessibilidade aos gestores das PME e alguma dificuldade em ter acesso às pessoas dos níveis técnico e administrativo.

No início de cada entrevista foram explicados os objetivos da pesquisa, bem como apresentados os temas definidos no roteiro construído, ficando os entrevistados livres para comentá-los segundo a sua disposição e sua compreensão. Além disso, buscando evitar qualquer aspecto de insegurança e de desconforto para os entrevistados quando da identificação das empresas onde trabalhavam e de si mesmos, foi assegurado o completo sigilo de identidade de ambos. Isso foi conseguido através da substituição do nome jurídico da empresa por uma letra do alfabeto (empresa $A ; B ; C$ ), enquanto que para o entrevistado foram criados identificadores (GR1 = gerencial; GR2 = técnico).

Os entrevistados têm mais de quatro anos de serviçosna PME associada. Em termos de faixa etária, observou-se que as idades dos entrevistados variavam de 25 a 58 anos. Quanto à escolaridade dos entrevistados, dois têm mestrado em administração, um tem mestrado em economia, um é bacharel em sistemas de informação e um entrevistado é graduando em administração de empresas.

\subsection{Definição domodelo de análise}

A busca de resultados na enquete campal visava associar os fatores organizacionais vis-à-vis o emprego de tecnologia e fora previsto surgir em termos de domínios de análise. Cada domínio seria associado a um 
fator organizacional incialmente previsto no escopo dos modelos de gestão de conhecimento ilustrados na seção 2.

Assim procedendo, ao lado da identificação das tecnologias em uso e seu direcionamento, poder-se-iam erguer categorias emergentes do campo, atreladas aos fatores organizacionais identificados, valendo-se da estratégia da análise proposicional de conteúdo temática (BARDIN, 2011), pela colagem de excertos emergentes que se associassem aos domínios previstos, quais sejam: estrutura; tratamento informacional; estratégias; processos e pessoas, todos mimetizados em apetrechos tecnológicos. Tais domínios serão detalhados mais adiante.

A seguir descortina-se a apuração dos resultados obtidos.

\section{Análise dos resultados da pesquisa}

Tem-se neste item o objetivo de mostrar a análise dos resultados obtidos na pesquisa. Aqui é ressaltado que a pesquisa completa abordou empresas dos segmentos comércio (4 empresas), indústria (4 empresas) e serviços (4 empresas); focalizando o presente artigo apenas no último segmento. Buscou-se, ao longo da análise, aproveitar as contribuições de cada um dos ouvidos, procurando observar aspectos de similitudes, complementaridades e distinções entre as diversidades de percepções. Ademais, fez-se um esforço no sentido de agrupar e evidenciar as opiniões dos entrevistados de acordo com o tema e não de acordo com o respondente. Por conseguinte, para uma mesma categoria de análise, é possível encontrar os comentários de mais de um respondente.

Os resultados são apresentados tomando por base os domínios sugeridos e que emergiram dos próprios dados. Desconsiderou-se, por não terem sidos identificados na realidade das PME de serviços os domínios estrutura e tratamento informacional que também representam fatores organizacionais obstativos ao uso da tecnologia da informação para a gestão do conhecimento nas PME de comércio e indústria. Em outros termos, para as PME de serviços ratificaram-se três os fatores organizacionais obstativos ao uso da TI para a GC: estratégias, processos e pessoas.

Entretanto, a análise far-se-á em primeiro plano quanto ao uso de TI nas PME.

\subsection{Utilização da tecnologia da informação pelas pequenas e médias empresas de serviços}

As tecnologias da informação destacadas na parte empírica do estudo,a partir de sua detecção em campo, foram enquadradas nos três grupos inicialmente postos: 
- TI para comunicação (TURBAN; RAINER; POTTER,2007) - Em termos de gestão do conhecimento, estas tecnologias permitem aos usuários o acesso ao conhecimento organizacional explícito, existente e necessário às suas atividades. Tal acesso acontece vale-se das ferramentas de comunicação de forma que são possíveis a aquisição, a transferência e o compartilhamento do conhecimento. Em campo destacaram-se o e-mail e a Internet;

- TI colaborativa(TURBAN; RAINER; POTTER,2007) - Essencialmente as contribuições das tecnologias da informação colaborativas para a gestão do conhecimento residem na possibilidade de transferência e de compartilhamento de conhecimentos explícitos entre pessoas pertencentes a grupos de trabalhos específicos e que apresentam uma tarefa ou objetivo em comum. Além disso, estas tecnologias permitem a transferência e compartilhamento de conhecimentos explícitos sobre os fluxos de trabalhos (documentos, tarefas) de um usuário a outro. No segmento a existência de artefatos deste naipe de tecnologias foi irrisória;

- TI para armazenamento(INMON, 2002) -Em geral elementos deste tipo de tecnologia emergem em termos de bancos de dados e sistemas de informação, o que faz esta TI contribuirpara a transferência e compartilhamento de conhecimentos explícitos no processo de gestão do conhecimento. Em campo surgiram citações a diversos sistemas e até mesmo à planilha Excelß.

Desse modo, a análise das questões referentes ao uso da TI, foi identificado o uso nas PME pesquisadas do segmento de serviços, das tecnologias da informação constantes no Quadro 2.

Como inequívoco destaque, as empresas de serviços pesquisadas utilizam bastante a Internet como meio de comunicação para pesquisa e como outro canal alternativo de propaganda e acesso a potenciais clientes.

Também se evidencia o maciço uso do e-mail para comunicação institucional externa e interna. O irrisório uso de tecnologias colaborativas e o diversificado uso e entendimento de tecnologia de armazenamento foi outro destaque encontrado na recolha de dados do segmento.

Quadro 2 Uso das tecnologias da informação pelas pequenas e médias empresas de serviços. 


\begin{tabular}{|c|c|c|c|c|}
\hline $\begin{array}{c}\text { PMES } \\
\text { PESQUISADAS }\end{array}$ & $\begin{array}{c}\text { TIS } \\
\text { CoMUNCATIVAS }\end{array}$ & $\begin{array}{c}\text { TIS } \\
\text { COLABORATIVAS }\end{array}$ & $\begin{array}{c}\text { TIS DE } \\
\text { ARMAZENAMENTO }\end{array}$ & OUTRAS \\
\hline Empresa A & $\begin{array}{l}E \text {-mail/ Intemet } \\
\text { Site Institucional }\end{array}$ & & $\begin{array}{l}\text { Sistema de controle } \\
\text { aca dêmico }\end{array}$ & $\begin{array}{l}\text { Sistemas de } \\
\text { folha de } \\
\text { pagamentos; } \\
\text { controle } \\
\text { contábil- } \\
\text { financeiro } \\
\end{array}$ \\
\hline Empresa E & E-mail / Intemet & & $\begin{array}{l}\text { Servidores com } \\
\text { acervo eletrônico } \\
\text { do que é produzido } \\
(\text { backup) }\end{array}$ & $\begin{array}{l}\text { Sistema } \\
\text { especifico de } \\
\text { editoração e } \\
\text { impressão de } \\
\text { apostilas e livros. } \\
\text { Sistema de } \\
\text { monitoramento } \\
\text { dos processos }\end{array}$ \\
\hline Empresa G & $\begin{array}{l}\text { MSN e Skype; } \\
\text { E-mail / Intemet }\end{array}$ & $\begin{array}{l}\text { Conferência via } \\
\text { telefone }\end{array}$ & $\begin{array}{lr}\text { Ferramenta } & \text { tipo } \\
\text { repositónio } & \text { que } \\
\text { padroniza } & \text { e } \\
\text { gerencia o acesso } \\
\text { compartilhado dos } \\
\text { funcionários aos } \\
\text { projetos } \\
\text { empresa }\end{array}$ & $\begin{array}{l}\text { Sistema de apoio } \\
\text { (Mantes) que } \\
\text { registra todo o } \\
\text { atendimento ao } \\
\text { cliente, projetos, } \\
\text { correções e até } \\
\text { melhorias }\end{array}$ \\
\hline Empresa L & $\begin{array}{l}\text { E-mail } \\
\text { Intemet } \\
\text { Tecnologia } \mathrm{WiFi} \\
\text { das operadoras de } \\
\text { telefonia }\end{array}$ & & $\begin{array}{l}\text { Planilhas } \\
\text { eletrônicas } \\
\left(\text { Excel }^{8}\right) ; \\
\text { banco de dados dos } \\
\text { sistemas de } \\
\text { informação }\end{array}$ & $\begin{array}{l}\text { ERP da } \\
\text { Microsiga; } \\
\text { Microsoft } \\
\text { CRM }\end{array}$ \\
\hline
\end{tabular}

Fonte: Dados coletados na pesquisa de campo.

A empresa A (faculdade de ensino superior privado) utiliza bastante a Internet como recurso de pesquisa para os seus docentes e discentes, além de permitir aos gestores pesquisarem sobre os seus concorrentes (datas de vestibulares, datas de realização de congressos, cursos de graduação e pós-graduação oferecidos etc.). Mesmo sendo a empresa mais antiga (69 anos) e a maior dentre as PME de serviços pesquisadas, foi observado um nível de uso da TI bastante limitado em que as diversas áreas usam sistemas diferentes, faltando uma arquitetura de tecnologia que venha fazer a integração das informações utilizadas nas diferentes áreas.

O site institucional permite que o docente disponibilize aos alunos todo o material didático do semestre letivo, além de permitir que o público em geral possa acessar a grade curricular dos cursos, possa conhecer os cursos de extensão oferecidos, o corpo docente e o conteúdo programático e ter conhecimento de quais são as atividades sociais de apoio à comunidade que são desenvolvidas.

O sistema de controle acadêmico realiza todo armazenamento das frequências dos alunos, histórico escolar, grade curricular a que os alunos têm vínculo, pagamento das mensalidades. Os sistemas de folha de 
pagamento e controle contábil-financeiro são responsáveis por controlar o fluxo de informações internas à organização como salários, férias, horas extras, pagamento de fornecedores e faturamento.

A empresa $E$ (prestadora de serviços de gráfica e editoração) também utiliza a TI de uma forma limitada, porque sendo a extensão recente de outro negócio (venda de máquinas para gráficas), os diretores ainda não têm experiência no novo segmento e não parecem estar priorizando-a em termos de investimento.

A Internet via e-mail é utilizada para a comunicação com os clientes e o recebimento dos pedidos de produção (apostilas e livros). Com a chegada desses pedidos é acionado o sistema que faz o cálculo de projeção de prazo de entrega e os sistemas específicos para editoração e impressão do material encomendado.

Há ainda um sistema que permite tanto ao gestor de produção quanto ao cliente (em sua residência) fazer o monitoramento dos processos. Tudo o que é produzido tem um backup (redundância) tanto em um acervo físico quanto em um acervo eletrônico que fica armazenado em servidores.

A empresa $G$ (como prestadora de serviços de desenvolvimento de software voltado para o varejo) ao contrário das outras duas PME do mesmo segmento utiliza mais recursos da tecnologia da informação. Além do correio eletrônico, como ferramentas de comunicação e integração dos escritórios são utilizadas o MSN Messenger $®$ - MSN e o Skype ${ }^{\circledR}$ para agendamento e realização de reuniões técnicas pela sua praticidade e agilidade no uso. A depender da complexidade de um projeto e da necessidade de mais troca de experiências ou colaboração entre os analistas e gestores há o uso de conferências via telefone.

Quanto ao processo de armazenamento, a empresa $G$ utiliza-se de um repositório que padroniza e gerencia o acesso compartilhado dos funcionários aos projetos da empresa, permitindo o desenvolvimento de vários projetos simultâneos e também permitindo que se tenha de forma organizada uma configuração de cada um de seus clientes que totalizam sessenta.

Ainda com relação à empresa $G$, é utilizado um sistema de apoio (Mantes) que registra todo o atendimento ao cliente, projetos, correções e até melhorias. Isto permite à empresa um acúmulo significativo de knowhow sobre os mais diversos aspectos envolvidos no desenvolvimento de software voltado ao varejo.

Finalmente, a empresa $L$ (prestadora de serviço de tecnologia da informação e engenharia) apresenta uma equipe de vendas espalhada pela região Nordeste, que se comunica e acessa à distância, via Internet, usando wifi das empresas operadoras de telefonia, às informações sobre os clientes que estão nos bancos de dados da empresa. O controle e o 
armazenamento de processos e oportunidades são feitos usando a planilha eletrônica Excel ${ }^{\circledR}$. Há também sistemas de informação que são: um aplicativo de administração de relacionamento com o cliente, CustomerRelationship Management - CRM, um sistema que está ligado à área administrativa e operacional, além da recente implantação de um sistema que vai absorver os outros dois e que fará o planejamento dos recursos empresariais, isto é, um sistema tipo Enterprise Resource Planning - ERP.

Neste segmento, a empresa L juntamente com a empresa $G$, foram consideradas as PME que mais utilizam recursos da tecnologia da informação quando comparadas às empresas $A$ e $E$ do mesmo segmento. Também ficou constatado que as PME de serviços usam a TI para as atividades operacionais diárias e dão ênfase à Internet como canal alternativo de propaganda e acesso a potenciais clientes e e-mail para comunicação e agilidade na transferência de informações.

No Quadro 3 são apresentadas as semelhanças e diferenças de uso da TI entre as PMEdo segmento de serviços observadas como casos nesta pesquisa.

Quadro 3Aspectos semelhantes e diferentes de uso da tecnologia da informação pelas pequenas e médias empresas de serviços.

\begin{tabular}{|c|c|}
\hline $\begin{array}{c}\text { ASPECTOS SEMELHANTES DE USO DA TI } \\
\text { ENTRE AS PMES }\end{array}$ & $\begin{array}{l}\text { PMES DE } \\
\text { SERVICOS }\end{array}$ \\
\hline $\begin{array}{l}\text { Intemet como canal altemativo de } \\
\text { propaganda e acesso a potenciais clientes } \\
\text { E-mail para comunicação e agilidade na } \\
\text { transferência de informações }\end{array}$ & $\begin{array}{l}\text { Empresa A } \\
\text { Empresa E } \\
\text { Empresa G } \\
\text { Empresa L }\end{array}$ \\
\hline $\begin{array}{c}\text { ASPECTOS DIFERENTES DE USO DA TI } \\
\text { ENTRE AS PMES }\end{array}$ & $\begin{array}{l}\text { PMES DE } \\
\text { SERVICOS }\end{array}$ \\
\hline Sistema de controle aca dêmico & Empresa $\mathbf{A}$ \\
\hline $\begin{array}{l}\text { Sistema de monitoramento dos processos de } \\
\text { produção (apostilas e livros) }\end{array}$ & Empresa E \\
\hline $\begin{array}{l}\text { Repositónio de ammazenamento, } \\
\text { padronização e gerenciamento de acesso } \\
\text { compartilhado aos projetos da empresa } \\
\text { Sistema de apoio (Mantes) aos diversos } \\
\text { aspectos envolvidos no deservolvimento de } \\
\text { software }\end{array}$ & Empresa G \\
\hline $\begin{array}{l}\text { Sistema de comunicação e acesso remoto } \\
\text { (WiFi) ao banco de dados da empresa } \\
\text { ERP }\end{array}$ & Empresa L \\
\hline
\end{tabular}

Fonte: Dados coletados na pesquisa de campo.

\subsection{Fatores organizacionais obstativos ao uso da tecnologia da informação para a gestão do conhecimento}


No segmento de serviços, asestratégias são marcadas pela inadequação dos recursos disponíveis à alta administração e que mesmo assim, são mal alocados e utilizados para o alcance dos objetivos básicos (MORAES, 2005). Ratificou-se em campo que há uma incapacidade de gerar capital excedente, para novos investimentos que permitam melhorias no portfólio de serviços e um crescimento sadio e planejado e também dificuldade no planejamento, análise e controle financeiro, de orçamentos e custos. Ademais, a TI é utilizada mais como suporte às atividades administrativas, comunicação com alguns clientes e armazenamento de projetos ou serviços realizados. As poucas estratégias que existem não enfatizam a gestão do conhecimento.

Além disso, nas PME, a definição de estratégias é feita exclusivamente a partir da observação e impressões particulares dos proprietários-gerentes, isto é, de sua intuição e perfil pessoal, o que dificulta desenvolver qualquer tipo de análise informacional fundamentada de seu ambiente (clientela, concorrência, tendências no segmento). Em tese: a estratégia é a orientação do proprietário. Por conseguinte, não foi percebido o uso eficiente dos métodos e ferramentas oferecidos pela TI para melhor planejar, desenvolver e dar suporte aos processos de utilização das informações coletadas do ambiente externo para a melhor definição e decisão de estratégias a serem adotadas. Apesar disso, o uso cotidiano da TI é visto como contributivo à vantagem competitiva organizacional, assim como à gestão da informação por permitir a previsibilidade, a eficiência e a qualidade informacional. No que diz respeito à possibilidade de gestão do conhecimento, a TI contribui para uma melhor formatação e uso do conhecimento organizacional existente.

Em termos de processos, as PME de serviços aqui alcançadas apresentaram uma centralização de diversas atividades e decisões nas mãos dos dirigentes, fato pitoresco e quase unânime em relação à prática de gestão em PME. Há ausência de regras e normas escritas levando a uma falta de definição clara de tarefas e funções. Faltam processos que possibilitem reconhecer a origem dos problemas e presta-se mais atenção em reclamações imediatas e visíveis. Há forte enfoque nos clientes externos em detrimento da organização das atividades, dos processos internos e do planejamento formal. Inexiste ainda a preocupação com a definição clara de tarefas e funções, nem com o estabelecimento de regras e normas escritas, também subutilizando as TI porque elas são usadas rotineiramente apenas para o controle de processos administrativos internos, comunicação com poucos clientes e fornecedores - mas não como auxílio no fluxo de trabalho através de seus recursos de comunicação, colaboração e armazenamento. Neste segmento também não foram identificados processos relacionados à criação, aquisição, codificação, transferência e compartilhamento de conhecimento. 
Por último, no fator pessoas, existem aspectos como o número reduzido de pessoas que se envolvem com o desenvolvimento de produtos e serviços, além da forte influência pessoal dos proprietários-dirigentes com excessiva centralização, traço quase existencial do segmento PME. Há ainda o aspecto da quase inexistência de treinamentos e atualizações para os funcionários, existindo entre estes competição e disputa para agradar aos gestores devido à proximidade entre os integrantes das áreas. A TI apenas auxilia as pessoas a melhor executar os processos administrativos internos, facilita a comunicação com os representantes externos, clientes, parceiros e fornecedores. Tudo isso, impossibilita qualquer esforço de gerenciar o conhecimento.

A identificação nas PME, da presença de um estilo de gestão centralizador, ineficaz, individualista, que incentiva a competição interna predatória, desvaloriza ou pouco reconhece as idéias e contribuições dos funcionários, não incentiva a criação, disseminação e o compartilhamento do conhecimento, refletindo aspectos culturais das PME, bastante personalizados na figura do líder, sem o necessário descolamento de papéis. Em realidade, esse estilo está subutilizando a tecnologia da informação e comprometendo o próprio processo decisório, porque em um ambiente organizacional com todas essas restrições não há geração ou tratamento qualitativo de informações úteis nem muito menos a possibilidade de sua gestão.

Ainda no fator organizacional pessoas, a TI foi considerada importante para o aperfeiçoamento, treinamento e integração das pessoas, o que contribui para o gerenciamento da informação uma vez que as pessoas percebem um maior grau de segurança e intercâmbio das informações. Não foi mencionada a contribuição da TI para a gestão do conhecimento porque na realidade vivenciada pelas PMEdo setor alcançado e da forma alcançada a gestão do conhecimento ainda não existe.

As empresas de serviços que apresentam algum destaque são as empresas G e L. Nestas duas, os recursos da TI são bem aproveitados porque a natureza de seus negócios tem na TI um elemento central. Essas PME apresentaram estratégias externas bem definidas, uso eficiente da TI, funções e processos bem delineados e pessoas capacitadas trabalhando em um ambiente desafiador. É perceptível a preocupação das áreas com o os processos de gerenciamento da informação (determinação da necessidade, obtenção, processamento, distribuição e utilização). Contudo, em nenhuma das duas empresas foi constatado a presença (no curto e médio prazo) da intenção de iniciar um projeto de gestão do conhecimento, apesar de ambos os gestores entrevistados reconhecerem a importância do recurso conhecimento para seus negócios. Com base na literatura, as empresas $\mathrm{G}$ e $\mathrm{L}$ apresentam condições atuais adequadas 
para desenvolverem a gestão do conhecimento. As demais PME do segmento de serviços que foram estudadas apresentam um gerenciamento informacional simples e mais voltado para o atendimento de necessidades internas.

\section{Considerações finais}

A tecnologia da informação tem possibilitado às organizações o desenvolvimento de uma nova dinâmica de competitividade e produtividade que consigam acompanhar as mudanças na sociedade da informação. A sua utilização permitiu a eficiente disponibilização e utilização de informações e conhecimentos para a tomada de decisões estratégicas, táticas ou operacionais pelos gestores, a ponto de Ortega, Blanco e Vanti (2013) afirmarem que a tecnologia da informação está revolucionando como as organizações e as pessoas estão fazendo negócios e como os gerentes usam os recursos intangíveis nos processos de tomada de decisão, planejamento e controle. Assim, pode-se dizer que a gestão de recursos intangíveis requer uma nova compreensão e esforço de como relacionar melhor os fatores organizacionais como estrutura, estratégia, processos, pessoas e tecnologia. É a este contexto que as empresas de pequeno e médio porte precisam estar atentas.

A pesquisa realizada permitiu notar a importância do uso da tecnologia da informação para uma integração maior dos fatores organizacionais e para a minimização da incerteza, da imprecisão e do improviso. A TI, como ferramenta, tem como elementos basilares e facilitadores dessa integração, a informação e o conhecimento. A busca pela identificação e o reconhecimento do papel desses recursos para o alcance da eficiência organizacional e da vantagem competitiva mostraram a necessidade da existência de novos modelos de gestão, o que pode ser demonstrado pelos diversos estudos teóricos $e$ aplicados(NONAKA; TAKEUCHI, 1997; LEONARD-BARTON, 1998; TERRA, 2001; ANGELONI, 2002; PROBST; RAUB; ROMHARDT, 2002; BEZERRA; BARBOSA; ESPEJO, 2013; PRADO et al., 2013; STEFANO et al., 2014).

O que caracteriza esses modelos, em termos gerais, é a centralidade que a informação e o conhecimento desempenham como recursos vitais para a organização moderna solucionar os seus problemas e ampliar a sua capacidade de inovação, uma vez que o seu compartilhamento possibilita às organizações basear-se em experiências passadas e conhecimentos adquiridos, para responder mais rapidamente aos desafios, desenvolver novas ideias e evitar reinventar a roda ou repetir erros vividos (RÊGO; FONTES FILHO; LIMA, 2013). Neste sentido a TI tem papel fundamental de apoio (CARVALHO, 2003; BEAL, 2004; LAUDON; LAUDON, 2016). 
Percebe-se ainda que estes modelos observam o esforço de gerenciamento da informação e do conhecimento, tendo a TI como suporte, consideram a singularidade de um fator organizacional bastante relevante ao êxito dessas iniciativas: as pessoas. São as pessoas que através de suas crenças, interpretações, imagens ou símbolos compartilhados e usados, que, em última análise, definirão e determinarão os aspectos de valor, acessibilidade, qualidade, confiabilidade e usabilidade da informação e do conhecimento.

Em termos empíricos, constatou-se na realidade vivenciada pelas PME que a gestão do conhecimento ainda não existe, a gestão da informação é perceptível em alguns casos e contribui para um potencial projeto futuro de GC. Contudo, o uso da TI ainda é essencialmente operacional e com fins de organizar, sistematizar e tornar eficientes as atividades cotidianas, estando mui longe da gestão do conhecimento. Isso acontece porque pela conjectura organizacional das PME há os fatores organizacionais estratégias, processos e pessoas que obstam isto, incapacitando o uso do conhecimento desenvolvido nas organizações como fator de diferencial competitivo (LINDERMAN; SCHROEDER; SANDERS, 2010; GOPAL; JOY, 2011; CHRISTOPHER; TANWAR, 2012).

Apesar de fornecer elementos contextualizados e a possibilidade de rica interpretação de fenômenos (COOPER; SCHINDLER, 2003), a abordagem qualitativa sofre críticas por carecer de capacidade para validação e generalização (KAPLAN; DUCHON, 1988), constituindo esta uma limitação da presente pesquisa. Além disso, pela natureza e métodos adotados, tornam-se inevitáveis a limitação cognitiva individual e a dimensão subjetiva empregadas para interpretar o fenômeno. A esta crítica a pesquisa também não pode escapar. Como sugestão de estudos futuros, poder-se-ia vislumbrar um esforço investigativo para pesquisar os fatores organizacionais obstativos ao uso da tecnologia da informação para a gestão do conhecimento em pequenas e médias empresas de outras regiões do nordeste edo restante do Brasil (norte, sul, sudeste e centro-oeste), comparando-os entre si e propondo alternativas de soluções para as especificidades de cada região.

\section{Referências}

ALBERTIN, A.L. Valor estratégico dos projetos de tecnologia da informação. Revista de Administração de Empresas, São Paulo, v.41, n.3, p.42-50, jul./set. 2001.

ALBANO, C.S. Adoção de novas tecnologias da informação: um estudo de problemas e ações nas cooperativas agropecuárias do Rio Grande do Sul. In: XXV Encontro Nacional da ANPAD, 2001, São Paulo. Cdrom...Campinas: ANPAD, 2001. 
ANGELONI, M.T. (coord.). Organizações do conhecimento:infra-estrutura, pessoas e tecnologia. São Paulo: Saraiva, 2002.

ANGUS, J.; PATEL, J.; HARTY, J. Knowledge management: great concept...but what is it? Information Week, March 1998.

AUDY, J.L.N.; ANDRADE, G.K. de; CIDRAL, A. Fundamentos de sistemas de informação. Porto Alegre: Bookman, 2005.

BARDIN, L. Análise de conteúdo. São Paulo: Edições 70, 2011.

BEAL, A. Gestão estratégica da informação. São Paulo: Atlas, 2004.

BEIJERSE, R.P. Knowledge management in small and medium-sized companies: knowledge management for entrepreneurs.JournalofKnowledge Management, v.4, n.2, p.162-179, 2000.

BEZERRA, C.A.; BARBOSA, S.R.; ESPEJO, M.M.S.B. Atividades de gestão do conhecimento entre organizações de assessoramento empresarial de naturezas distintas: um estudo em empresas no estado do Paraná.

Perspectivas em Gestão \& Conhecimento, João Pessoa, v. 3, n. 2, p. 112130, jul./dez. 2013.

BORTOLI NETO, A. de. Tipologia de problemas das pequenas e médias empresas. 1980. Dissertação. (Mestrado) - Universidade de São Paulo.

CALADO, M.A.F. Administração empreendedora: da ideia ao plano de negócio. Recife: Livro Rápido, 2004.

CARVALHO, R.B. Tecnologia da informação aplicada à gestão do conhecimento. Belo Horizonte: C/Arte, 2003.

CHRISTOPHER, D.; TANWAR, A. Knowledge management in outsourcing environment: people empowering people. The IUP Journal of Knowledge Management, v. 10, n. 2, p. 61-86, 2012.

COOPER, D.R.; SCHINDLER, P.S. Métodos de pesquisaemadministração. Porto Alegre: Bookman, 2003.

CUNHA, J.A.C.; YOKOMIZO, C.A.; CORRÊA, H.L.; PASSADOR, J.L. A evolução do trabalho baseado em conhecimentos e competências na economia contemporânea. In: CONGRESSO INTERNACIONAL DE GESTÃO DA TECNOLOGIA E SISTEMAS DE INFORMAÇÃO, 5, 2008, São Paulo. Cdrom...São Paulo: 2008.

DAVENPORT, T.Enterprise analytics: optimize performance, process, and decisions through big data.Upper Saddle River, New Jersey: FT Press Operations Management, 2012.

DAVENPORT, T.; PRUSAK, L. Conhecimentoempresarial:como as organizaçõesgerenciam o seu capital intelectual. Tr. Lenke Peres. Rio de Janeiro: Campus, 1998. 
DI MARTINO, B. et al. Big data (lost) in the cloud. International Journal of Big Data Intelligence, v. 1, n.1/2, p.3 - 17, 2014.

EGBU, C.O.; BOTTERILL, K. Knowledge management and intellectual capital: benefits for project based industries. In: KELLY, J., HUNTER, K. (eds). Proceedings of the RICS Foundation - construction and building research conference (COBRA). Glasgow Caledonian University, 3-5 september, p.414-22, 2001.

EVAN, P.B.; WURSTER, T.S. Strategy and the new economics of information. Boston: Harvard Business Review, v.75, n.5, p.70-82, set./out., 1997.

FELL, A.F.A. Fundamentos da gestão do conhecimento. Recife: Ed. Universitária da UFPE, 2011.

FELL, A.F.A. Análise dos fatores organizacionais obstativos ao uso da tecnologia da informação para a gestão do conhecimento: uma realidade vivenciada em pequenas e médias empresas da Região Metropolitana do Recife. 2009. Tese. (Doutorado) - Universidade Federal de Pernambuco, Recife, 2009.

FELL, A.F.A.; RODRIGUES FILHO, J.; OLIVEIRA, R.R. Um estudo da produção acadêmica nacional sobre gestão do conhecimento através da teoria do conhecimento de Habermas. São Paulo, Revista de Gestão da Tecnologia e Sistemas de Informação, v. 5, n. 2, p. 251-268, 2008.

FELL, A.F.A. Análise do comércio eletrônico (CE) em pequenas e médias empresas (PMEs) da Região Metropolitana do Recife (RMR) - barreiras e obstáculos.2003. Dissertação. (Mestrado) - Universidade Federal de Pernambuco), Recife, 2003.

FREITAS JUNIOR, J.C.S.; MAÇADA, A.C.G. Processo decisório no contexto de Big Data: estudo de caso em uma empresa de varejo. In: SEMEAD, 17- FEA-USP, São Paulo.Anais [...] 2014.

FREIRE, P.S.; SPANHOL, F.J. Conhecimento organizacional: produto ou processo? Perspectivas em Gestão \& Conhecimento, João Pessoa, v. 4, n. 1, p. 3-21, jan./jun. 2014.

FREY, R.S. Knowledge management, proposal development, and small businesses. Journal of Management Development, v. 20, n.1, p.38-54, 2001.

GELINAS, R.; BIGRAS, Y. The characteristics and features of SMEs: favorable or unfavorable to logistics integration? Milwaukee, JournalofSmall Business Management, v.42, n.3, Jul. 2004. 
GONZALEZ, R. V. D.; MARTINS, M. F.; MELO, T. M. Gestão do conhecimento: tipologia a partir dos fatores contextuais da organização. Transinformação, v. 30, n. 2, p. 249-266, 2018.

GOPAL, C. S. R.; JOY, P. A. Creation of knowledge management system.Advances in Management, v. 4, n. 11, p.7-14, Nov. 2011.

HARRISON, D.; MYKYTYN, P.; RIEMENSCHNEIDER, C. Executive decisions about information technology and competitive strategy in small business: theory and empirical tests. Information Systems Research, v.8, n.2, p.171-195, 1997.

HAUTALA, J. International academic knowledge creation and ba. A case study from Finland. Knowledge Management Research \& Practice, v. 9, n. 1, p. 4-16, 2011.

HENG, M.S.H. Mapping intellectual capital in small manufacturing enterprise. Journal of Intellectual Capital, v. 20, n.1, p.53-60, 2001.

HUANG, L. S.; QUADDUS, M.; ROWE, A. L.; LAI, C. P. An investigation into the factors affecting knowledge management adoption and practice in the life insurance business. Knowledge Management Research\&Practice, v. 9, n. 1, p. 58-72, 2011.

IBGE - INSTITUTO BRASILEIRO DE GEOGRAFIA E ESTATÍSTICA Estatísticas do Cadastro Central de Empresas. Brasília, 2010.

INMON, W. Building the data warehouse. New Jersey: John Wiley and Sons, 2002.

JACKSON, C. Process to product - creating tools for knowledge management. Conference in Lisbon, Portugal, 1999.

KAHNEMAN, D., LOVALLO, D.; SIBONY, O. Before You Make That Big Decision. Harvard Business Review, v. 89, n.6, p. 50-60, 2011.

KAMARA, J.M., ANUMBA, C.J., CARRILLO, P.M. A Clever approach to selecting a knowledge management strategy. International Journal of Project Management, v.20, n.3, p.205-211, 2002.

KAPLAN, B.; DUCHON, D. Combining qualitative and quantitative methods in information systems research: a case study. MIS Quarterly, v.12, n.4, 1988.

KASSAI, S. As empresas de pequeno porte e a contabilidade. 1996. Dissertação. (Mestrado) -Universidade de São Paulo. 1996.

KAUTZ, K.; THAYSEN, K. Knowledge, learning and IT support in a small software company. Journal of Knowledge Management, v. 5, n.4, p.349$357,2001$. 
KESS, P.; LAW, K.M.Y.; KANCHANA, R.; PHUSAVAT, K. Critical factors for an effective business value chain. Industrial Management and Data Systems, v.110, n.1, p.63-77, 2010.

KISSIMOTO, K.O. A influência da tecnologia da informação na estratégia de personalização nas empresas brasileiras. 2011. Dissertação.

(Mestrado) - Universidade de São Paulo, São Paulo, 2011.

KRUGLIANSKAS, I.; TERRA, J.C.C. Gestão do conhecimento em pequenas e médias empresas. 2ed. São Paulo: NegócioEditora, 2004.

LAUDON, K.C. ; LAUDON, J.P. Management Information

Systems:managing the digital firm. 14. ed. New York: Person, 2016.

LAURINDO, F.J.B. ; CARVALHO, M.M. ; PESSÔA, M.S.P.; SHIMIZU, T.

Selecionando uma aplicação de tecnologia da informação com enfoque na eficácia: um estudo de caso de um sistema para PCP. São Carlos, Revista Gestão e Produção, v.9, n.3, p.377-396, dez. 2002.

LEONARD-BARTON, D. Nascente do saber: criando e sustentando as fontes de inovação. Rio de Janeiro: Fundação Getúlio Vargas, 1998.

LEONARDI, J.; BASTOS, R.C. Bases epistemológicas da teoria de criação de conhecimento organizacional. Perspectivas em Gestão \& Conhecimento, v. 4, n. 2, p. 3-18, 2014.

LIM, D. ; KLOBAS, J. Knowledge management in smallenterprises. The Electronic Library, v.18, n.6, p.420-432, 2000.

LINDERMAN, K.; SCHROEDER, R. G.; SANDERS, J. A knowledge framework underlying process management. DecisionSciences, v. 41, n. 4, p. 689-719, Nov. 2010.

LUNARDI, G. L.; DOLCI, P. C. ; MAÇADA, A. C. G. Adoção de tecnologia de informação e seu impacto no desempenho organizacional: um estudo realizado com micro e pequenas empresas. São Paulo,Revista de Administração da USP, v.45, n.1, p.05-17, jan./mar. 2010.

MAGNIER-WATANABE, R.; BENTON, C. ; SENOO, D. A study of knowledge management enablers across countries. Knowledge Management Research \& Practice, v. 9, n. 1, p. 17-28, 2011.

MARCHAND, D.A.; KETTINGER, W.J.; ROLLINS, J.D.

Desempenhoempresarial e gestão da informação: a visão do topo. In: DAVENPORT, T.H.; MARCHAND, D.A.; DICKSON, T. Dominando a gestão da informação. Porto Alegre: Bookman, 2004.

MARTINS, G.A. Manual para elaboração de monografias. 2 ed. São Paulo: Atlas, 1994.

MCGEE, J. V.; PRUSAK, L. Gerenciamento estratégico da informação:aumente a competitividade e eficiência de sua empresa 
utilizando a informação como uma ferramenta estratégica. Rio de Janeiro: Elsevier, 1994.

MERRIAM, S. What is qualitative research? In: Qualitative research and case study applications in education. San Francisco: Jossey-Bass, 1998.

MIAKE, A.H.S.; CARVALHO, R.B; PINTO, M. Gestão do conhecimento do cliente (CKM): interfaces e sinergias entre a gestão do relacionamento com clientes (CRM) e as ferramentas de gestão do conhecimento. In: SEMEAD, 17 - FEA-USP, São Paulo. 2014.

MORAES, G.D.A. A tecnologia da informação na pequena empresa: uma investigação sobre sua contribuição à gestão estratégica da informação nos empreendimentos industriais dos minidistritos de São José do Rio Preto - SP. São Paulo, 2005. Dissertação. (Mestrado) - Escola de Engenharia de São Carlos da Universidade de São Paulo, São Paulo, 2005. MORAES, G.D.A.; TERENCE, A.C.F.; ESCRIVÃO FILHO, E. A tecnologia da informação como suporte à gestão estratégica da informação na pequena empresa. Revista de gestão da tecnologia e sistemas de informação. v.1, n.1, p.28-44, 2004.

MORAES, G.D.A.; ESCRIVÃO FILHO, E. Agilizando as informações na pequena empresa: caracterização, obstáculos e vantagens do uso da tecnologia da informação. In: SEGET,17, Resende, 2004.

MURPHY, S.; SAMIR, W. 'In the cloud' it creates new opportunities for network service providers. JournalofTelecommunications Management [S.I.], v.2, n.2, p.107-120, 2009.

NGANGA, C.S.N.; LEAL, E.A. A utilidade de um sistema ERP (Enterprise Resource Planning) no processo de gestão de pequenas empresas. In: SEMEAD, 16 - FEA-USP, São Paulo. 2013.

NONAKA, I.; TAKEUCHI, H.Criação de conhecimento na empresa: como as empresas japonesas geram a dinâmica da inovação. Rio de Janeiro:

Campus, 1997.

ORTEGA, A. C. ; BLANCO, R. R.; VANTI, A. A. Information Management in Global Environments: SwarmIntellegence in

multilingualeconomicdocumentrepositories. Inf. \&Soc.:Est., João Pessoa, v.23, n.1, p. 27-38, jan./abr. 2013.

PRADO, H.A. ; NOBRE, M.K.; FERNEDA, E.; NEHME, C.C.; HEDLER, H.C. Sinergias entre gestão do conhecimento e planejamento tecnológico; um estudo de caso. Perspectivas em Gestão \& Conhecimento, João Pessoa, v. 3, n. 2, p. 92-111, jul./dez. 2013. 
PRAHALAD, C.K.; RAMASWAMY, V. O futuro da competição: como desenvolver diferenciais inovadores em parceria com os clientes. Rio de Janeiro: Campus, 2004.

PRATES, G.A.; OSPINA, M.T. Tecnologia da informação em pequenas empresas: fatores de êxito, restrições e benefícios. Revista de administração contemporânea, v.8, n.2, abr./jun. 2004, p.9-26.

PRATES, G.A. Tecnologia de informação em pequenas empresas: analisando empresas do interior paulista. Administração on-line, v.3, n.4, out./dez., 2002.

PROBST, G. ; RAUB, S.; ROMHARDT, K. Gestão do conhecimento: os elementos constitutivos do sucesso. Porto Alegre:Bookman, 2002.

QUINTAS, P. Knowledge management in the $21^{\text {st }}$ century. New York: Springer, 2002.

QUINTAS, P.; LEFRERE, P.; JONES, G. Knowledge management: a strategic agenda. Long Range Planning, v.30, p. 385-391, 1997.

RÊGO, R. C. A. ; FONTES FILHO, J. R.. LIMA, D. F. P. Confiança organizacional e compartilhamento e uso do conhecimento tácito. RAERevista de Administração de Empresas, v. 53, n. 5, 2013.

ROESCH, S.M.A.Projetos de estágio e de pesquisa em administração.2ed. São Paulo: Atlas, 1999.

ROSÁRIO, C. R. ; KIPPER, L. M.; FROZZA, R. Técnicas de elicitação de conhecimento tácito: um estudo de caso aplicado a uma Empresa do Ramo Metalúrgico. Inf. \&Soc.:Est., João Pessoa, v.24, n.1, p. 117-134, jan./abr. 2014.

RUGGLES, R. Knowledge management tools. Oxford: ButterworthHeinemann, 1997.

SEDIGHI, M. M.; MOKFI, T.; GOLRIZGASHTI, S. Proposing a customer knowledge management model for customer value augmentation: A home appliances case study.Database Marketing \&CustomerStrategy Management. v. 19, pp. 321-347, 2012.

STEFANO, N.M.; FILHO, N.C.; FREITAS, M.C.D.; MARTINEZ, M.A.T. Gestão de ativos intangíveis: implicações e relações da gestão do conhecimento e capital intelectual. Perspectivas em Gestão \& Conhecimento, João Pessoa, v. 4 , n. 1 , p. 22-37, jan./jun. 2014.

SHAPIRO, C., VARIAN, H.R.A economia da informação. Rio de Janeiro: Campus, 1999.

SILVA, A.L. ; FISCHMANN, A.A. A adoção de tecnologia de informação em canais de distribuição. Revista de Administração, São Paulo, v.37, n.2, p.6-16, abr./jun., 2002. 
SILVA, V.L. Diagnóstico do nível de tecnologia da informação e dos sistemas de informação contábeis-gerenciais no processo decisório das micro e pequenas empresas do ramo de confecções do município de Colatina - ES. Florianópolis, 2002. Dissertação (Mestrado) -Universidade Federal de Santa Catarina. 2002.

SIQUEIRA, E.S.; SOUZA, C.A.; CHAGAS, V. Uso das TIC nas pequenas e médias empresas brasileiras: construção de um índice de uso utilizando dados da pesquisa TIC empresas e modelagem de equações estruturais. In: SEMEAD, 17 - FEA-USP, São Paulo. 2014.

SOUZA, . A.; ARPINO, G. TI e eficiência organizacional: um estudo no setor brasileiro de bens de capital mecânicos com foco em micro, pequenas e médias empresas. Produção, v. 21, n. 4, p. 742-754, out./dez. 2011.

SOUZA, C.A.A.; HENDRIKS, P.H.J. The diving bell and the butterfly: the need for grounded theory in developing a knowledge-based view of organizations.OrganizationalResearchMethods; v. 9, n. 3., P. 315-338,Jul, 2006.

STABILE, S. ; CAZARINI, E.W. Tecnologia de informação e os níveis gerenciais das organizações. In: SIMPEP - SIMPÓSIO DE ENGENHARIA DE PRODUÇÃO, 7, 2000. Bauru. Anais [...] São Paulo, 2000.

STAKE, R.E. Case studies. In: DENZIN, N.K., LINCOLN, Y.S. Handbook of qualitative research. California: SAGE, 1994.p. 237-247.

STOLLENWERK, M.F.L. Gestão do conhecimento: conceitos e modelos. In: TARAPANOFF, K. (org). Inteligência organizacional e competitiva. Brasília: Editora UNB, 2001.

SWAN, J.; SCARBROUGH, H. Knowledge management: concepts and controversies. Journal of Management Studies, 38, 913-921, 2001.

SWAN, J.; ROBERTSON, M., BRESNEN, M.Knowledge Management and the Colonization of Knowledge. CMS Conference, 2001.

SWAN, J.; SCARBROUGH, H.; PRESTON, J.Knowledge Management - The Next Fad to Forget People. In: PRIES-HEJE, J. et al. (Eds.)European Conference on Information Systems, 7., 1999. Copenhagen. Proceedings [...]. v.1-2, June 23-25. Copenhagen: 1999. p.668-678.

TEECE, D.J. Strategies for managing knowledge assets: the role of firm structure and industrial context. LongRage Planning, v. 33, p. 35-54, 2000.

TERRA, J.C.C.Gestão do Conhecimento: aspectos conceituais e estudo exploratório sobre as práticas de empresas brasileiras.In: FLEURY, M. T. L.; OLIVEIRA JR, M. de M. Gestão estratégica do conhecimento:integrando aprendizagem, conhecimento e competências.São Paulo: Atlas, 2001. 
THONG, J.Y.L. Resource constraints and information systems implementation in Singaporean small businesses. Omega, n.29, p.143156, 2001.

TRIVIÑOS, A.N.S. Introdução à pesquisa em ciências sociais: a pesquisa qualitativa em educação. São Paulo: Atlas, 1995.

TURBAN, E.; RAINER JR., K.; POTTER, R.E. Introdução a sistemas de informação. Rio de Janeiro: Elsevier, 2007.

TYNDALE, P. A taxonomy of knowledge management software tools: origins and applications. EvaluationandProgram Planning, v.25, p.183-190, 2002.

VIDAL, A.G.da R. Seleção de pacotes de software administrativo na pequena e média empresa - um estudo exploratório. São Paulo, 1990. Dissertação. (Mestrado), Universidade de São Paulo. São Paulo, 1990.

WALTON, R. Tecnologia da informação: o uso da TI pelas empresas que obtém vantagem competitiva. São Paulo: Atlas, 1993.

WENGER, E.Communities of practice: Learning, meaning and identity. Cambridge: Cambridge University Press, 1998.

WENSLEY, A. Tools for knowledge management. In: BPRC CONFERENCE ON KNOWLEDGE MANAGEMENT: concepts and controversies, Feb. 10-11, 2000. Coventry: University of Warwick, 2000.

WICKERT, A.; HERSCHEL, R. Knowledge management issues for smaller business. Journal of Knowledge Management, v. 5, n.4, p.329-337, 2001.

WILLERDING, I. A. V. et al. Indicadores relevantes para o sucesso do compartilhamento do conhecimento tácito nas empresas: evidências empírico-exploratórias em PMEs catarinenses. Revistagestão industrial, $v$. 11 , p. 202-228, 2015.

WONG, K.Y. Critical success factors for implementing knowledge management in small and medium enterprises. Industrial Management Data Systems. v.105, n.3, p.261-279, 2005.

XAVIER, R.O. Por uma nova postura gerencial baseada no uso de um sistema de gerenciamento do relacionamento com o cliente (CRM). Recife, 2003. Dissertação. (Mestrado). Universidade Federal de Pernambuco. Recife: 2003.

ZACK, M.H. Knowledgeandstrategy. Boston: Butterworth-Heinemann, 1999.

ZIMMERER, T. W.; SCARBOROUGH, N. M. Essentials of small business management. Macmillan College, 1994. 